\title{
ROLE OF B-MODE ULTRASOUND IN THE EVALUATION OF ORBITAL MASSES
}

\author{
Radiodiagnosis \\ Dr. Sushilkumar \\ Kamlakar Kale \\ MBBS, MD (Radiodiagnosis) Professor, Department of Radiodiagnosis, Mahatma \\ Gandhi Institute Of Medical Sciences, Sewagram, Wardha, Maharashtra, India.
}

\section{Dr. Atul Manohar} Dhok*

MBBS, MD (Radiodiagnosis) Assistant Professor, Department of Radiodiagnosis, Mahatma Gandhi Institute Of Medical Sciences, Sewagram, Wardha, Maharashtra, India. *Corresponding Author

\section{ABSTRACT}

Introduction: Blindness and Vision impairment cause significant morbidity amongst Indian population. According to WHO estimates, about 20.5 $\%$ people in India are categorized as blind while $22.2 \%$ people have low vision. The orbital masses owing to the small anatomical volume of orbit can cause devastating consequences. Ultrasound and colour doppler are useful in analysis of orbital masses with real time evaluation, excellent soft tissue resolution, localisation, and benign or malignant nature of masses. This study evaluates the role of ultrasound in orbital masses.

Aim: To study the role of ultrasound in evaluation and identification of orbital masses.

Study design \& settings: Prospective observational study in ultrasound unit of a rural hospital in central India.

Materials and methods: A total of 316 consenting patients were studied for 1 year using high frequency linear and curved probes. Statistical analysis was done using SPSS software (IBM Inc.)

Results: Out of the 316 patients, total 15 cases were identified to have orbital masses. Most common orbital mass lesions observed were lacrimal gland tumours. Most of the masses were extraconal in location and most common ultrasound appearance of the lesions was solid with most of the malignant masses showing vascularity on colour doppler imaging.

Conclusion: Ultrasound can identify and characterize orbital masses based on appearance and echotexture with accurate localisation. It is cheap and easily available with no radiation hazards Pre-operative ultrasound and Colour Doppler evaluation of orbit can help in predicting prognosis of the disease.

\section{KEYWORDS}

Original article, Ultrasound, Radiology, Ocular ultrasound, Colour Doppler, Ophthalmology, orbital masses, ophthalmic ultrasound.

\section{INTRODUCTION:}

Vision impairment cause significant morbidity amongst Indian population. According to $\mathrm{WHO}$ estimates, about $20.5 \%$ people in India are categorized as blind while $22.2 \%$ people have low vision.(1) B-scan ultrasonography has become an extremely important tool in diagnosis of orbital lesions, especially in cases of opaque light conducting media.(2)(3). The orbital masses, though rare, form a significant chunk of the orbital. The small anatomical volume of orbit can the cause of devastating consequences even with a small space occupying lesion in the orbital cavity.(4) Ultrasonic imaging of orbit has unique acoustical advantages because of the cystic structure and superficial location of the eye.

Colour Doppler imaging adds significant adjunctive benifits in diagnosis and evaluation of orbital pathologies. Colour Doppler can be used for the evaluation of normal orbital vessels anatomy, vascular flow characteristics of tumour and detection of vascular malformations such as arteriovenous fistulae. The application of colour Doppler imaging in ophthalmic ultrasound evaluation is relatively new and it has been used mostly in the evaluation of orbital tumours and conditions with altered orbital vascular hemodynamics.(5)(6).

Most common orbital symptoms due to orbital mass lesions are pain, redness, watering and proptosis. $(7,8)$. Orbital masses can be classified as orbital or extraorbital masses depending upon their location. In this study we have only included the orbital masses for analysis. Orbital masses can further be classified according to their location (Intraconal/Extraconal), invasive potential (benign/malignant), nature of the lesion (cystic/solid/mixed),vascularity (Vascular /avascular) and histological type (Vasculogenic / lymphoproliferative / Lacrimal gland/optic nerve/ peripheral nerve sheath) (9).

B-scan ultrasonography has become an extremely important tool in diagnosis of intra-ocular lesions, especially when the light conducting media opaque due to opacities of cornea, aqueous humour, lens or vitreous.(3,8). Possibility of dynamic studies with eye movements and real time imaging is an added advantage of B-scan in localization of lesions. The value of CT and MRI in the evaluation of orbital tumours or metastases cannot be undermined in todays scenario. However, considering the effects of radiation on a delicate structure such as eye and issue of higher cost of MRI imaging studies, the sonographic evaluation of eye as first line radiological investigation in the preoperative evaluation of orbital tumours is likely to help in surgical planning and prognostic analysis of the patient.

B-Mode ultrasound is thus an important clinical imaging tool for ocular pathologies. It is cheap, easy to perform, easy to interpret and more so without any patient discomfort. It is thus rapidly becoming preferred method in preoperative evaluation of ocular pathologies. With the advent of newer modalities such as CT and MRI, the sonographic evaluation of eye with Colour Doppler imaging can act as a useful adjunct and can help in postoperative follow up and prognostication of patient.

\section{AIMS AND OBJECTIVES:}

1) To identify and characterize various orbital masses on B-Mode and colour Doppler ultrasound scan

2) To study the use of Colour Doppler imaging in orbital masses and its complementarity with B-Mode ultrasound.

\section{MATERIALS AND METHOD:}

After clearance from the ethical committee of the institute. A total of 316 consenting patients (Total of 632 eyes) with ocular complaints were studied over a period of 1 year (1st January 2014 to $31 \mathrm{st}$ December 2015). Out of these 15 patients were found to have orbital masses. Patients of all age groups and both sexes with orbital complaints who were referred to our ultrasound unit. Patient with severe ocular trauma and immediate postoperative patient in which contact ultrasound scan is contraindicated.

1)Equipment Used: Phillips HD11 XE ultrasound machine with linear probe of frequency 5-12 MHz and curved probe of frequency 2-5 MHz. GE Voluson S6 ultrasound machine with linear probe of frequency upto $12 \mathrm{MHz}$ and curved probe of frequency up to $4 \mathrm{MHz}$. Statistical analysis was done using SPSS software (IBM Inc.)

OBSERVATIONAND RESULTS:

Table 1: Age wise distribution of patients

\begin{tabular}{|l|l|l|}
\hline Age Group(yrs.) & No of patients & Percentage (\%) \\
\hline $0-10$ yrs. & 15 & 4.75 \\
\hline $11-20$ yrs. & 20 & 6.33 \\
\hline $21-30$ yrs. & 30 & 9.49 \\
\hline $31-40$ yrs. & 22 & 6.96 \\
\hline $41-50$ yrs. & 43 & 13.61 \\
\hline
\end{tabular}




\begin{tabular}{|l|l|l|}
\hline $51-60$ yrs. & 59 & 18.67 \\
\hline $61-70$ yrs. & 88 & 27.85 \\
\hline $71-80$ yrs. & 33 & 10.44 \\
\hline $81-90$ yrs. & 6 & 1.90 \\
\hline Total & 316 & 100 \\
\hline Mean \pm SD & $50.24 \pm 20.36(1$ day to 85 years $)$ \\
\hline
\end{tabular}

Table 2: Gender wise distribution of patients

\begin{tabular}{|l|l|l|}
\hline Gender & No of patients & Percentage (\%) \\
\hline Male & 195 & 61.7 \\
\hline Female & 121 & 38.3 \\
\hline Total & 316 & 100 \\
\hline
\end{tabular}

Table 3: Spectrum of various orbital masses

\begin{tabular}{|l|l|l|l|}
\hline Orbital masses & $\begin{array}{l}\text { No. eyes } \\
(\mathbf{n = 1 5})\end{array}$ & $\begin{array}{l}\text { Percentage } \\
\mathbf{( \% )}\end{array}$ & $\begin{array}{l}\text { \% in total } \\
\text { patients } \\
(\mathbf{n = 3 1 6})\end{array}$ \\
\hline Lacrimal gland tumors & 3 & 20.00 & 0.94 \\
\hline Dermoid/ Lipodermoid & 2 & 13.33 & 0.63 \\
\hline Cystic granuloma & 1 & 6.66 & 0.35 \\
\hline Foreign body granuloma & 2 & 13.33 & 0.63 \\
\hline Retinoblastoma & 2 & 13.33 & 0.63 \\
\hline Rhabdomyosarcoma & 1 & 6.66 & 0.35 \\
\hline Hemangioma & 2 & 13.33 & 0.63 \\
\hline Metastases & 2 & 13.33 & 0.63 \\
\hline
\end{tabular}

Table 4: Distribution of orbital masses according to location.

\begin{tabular}{|l|l|l|}
\hline Location & Number of masses $(\mathbf{n}=\mathbf{1 5})$ & Percentage (\%) \\
\hline Intraconal & 3 & $20 \%$ \\
\hline Extraconal & 12 & $80 \%$ \\
\hline Total & 15 & $100 \%$ \\
\hline
\end{tabular}

Table 5: Distribution of mass lesions according to ultrasound characteristics.

\begin{tabular}{|l|l|l|}
\hline $\begin{array}{l}\text { Ultrasound } \\
\text { Characteristics }\end{array}$ & $\begin{array}{l}\text { No. Of mass lesions } \\
(\mathbf{n = 1 5})\end{array}$ & Percentage (\%) \\
\hline Solid & 12 & 80 \\
\hline Cystic & 2 & 13.33 \\
\hline Mixed & 1 & 6.66 \\
\hline Total & 15 & 100 \\
\hline
\end{tabular}

Table 6: Distribution of masses according to presence or absence of vascularity on Color Doppler Imaging.

\begin{tabular}{|l|l|l|}
\hline Vascularity of CDI & Number of masses (n=14) & Percentage (\%) \\
\hline
\end{tabular}

\begin{tabular}{|l|l|l|}
\hline Vascularity of CDI & Number of masses $(\mathbf{n}=\mathbf{1 4})$ & Percentage (\%) \\
\hline Present & 10 & $66.67 \%$ \\
\hline Absent & 5 & $33.33 \%$ \\
\hline Total & 15 & $100 \%$ \\
\hline
\end{tabular}

\section{DISCUSSION:}

The primary objective of the study was to assess the role of ultrasound in the evaluation of orbital masses, especially in the patients with opaque ocular media hindering fundoscopic evaluation. A total of 316 patients (total 632 eyes) were studied over a period of 1 year in our ultrasound unit. The age range of the examined patients was from 1 day to 85 years for both sexes with mean age ( \pm standard deviation) of $50.24( \pm 20.46)$ years. In this study, out of 316 patients studies 195 patients $(61.7 \%)$ were males and 121 patients $(38.3 \%)$ were females with male to female ratio of $1.6: 1$ which correlates well with the previous studies. $(3,7,8)$.

Out of the 316 patients studied, 15 patients were found to have various orbital masses, out of which 8 were malignant and 7 were benign Most of the orbital masses were found to be extraconal in location $(80 \%)$ and $66.67 \%$ showed vascularity on Doppler imaging. Most common ultrasound appearance was solid masses.

1) Lacrimal gland tumours:Most common orbital masses in our study were found to be lacrimal gland tumours all of which presented with proptosis. Most common orbital masses were lacrimal gland tumours $(20 \%)$ which comprised one each of pleomorphic adenoma, adenoid cystic carcinoma and squamous cell carcinoma. Most of the lacrimal gland masses appear acoustically solid with internal low resistance vascular flow on Doppler imaging. $(10,11)$

2) Dermoid or Lipodermoid: In our study we found one case each of conjunctival dermoid and Lipodermoid. Dermoid appears well defined cystic lesion with some internal solid hyperechoic area and showed no vascularity on Colour Doppler imaging. Lipodermoid appeared well defined hyperechoic conjunctival mass with no vascularity on Doppler imaging.(12)

3) Cystic granuloma: One case of cystic granuloma was identified in our study which appeared cystic with few internal echoes and no vascularity on Colour Doppler imaging. Both the lesions were found to be cholesterol granuloma which may mimic malignancy and tend to recur.(13)

4) Foreign body granuloma: The foreign body granulomas were found in 2 cases who had history of trauma. On ultrasound both the lesions were extraconal and appeared hypoechoic with central hyperechoic foreign body without any vascularity on colour doppler imaging.

5) Retinoblastoma: Retinoblastoma is usually cited as the commonest intraocular tumour in paediatric age group with the incidence of 1 in 20,000 births. It usually presents unilaterally but can also be seen bilaterally in $1 / 3^{\text {rd }}$ of cases. The most common presentation is leukocoria or an abnormal whitish reflection from the retina of the eye of affected child.(14) USG shows echogenic lesion and calcium deposits with optic nerve involvement in many cases. In our study 2 cases of unilateral retinoblastoma were identified. Both cases presented with history of leukocoria and proptosis. The lesions had solid appearance with numerous areas of internal calcification causing posterior acoustic shadowing. The axial length measurement is imperative in a patient with suspected retinoblastoma as it usually shows normal or increased axial length while the pathologies mimicking retinoblastoma usually show reduced axial length.(15). On Colour Doppler imaging low resistance flow was seen in the mass in both cases which signified actively growing malignancy. One of the patients had recurrence of the lesion within 2 years of enucleation.

6) Rhabdomyosarcoma: Rhabdomyosarcoma has been described as the commonest primary malignant orbital tumour of childhood in literature. (16) The tumour arises from an extraocular muscle and may involve any part of the orbit, but usually occurs in the superonasal quadrant and appears on ultrasound as a poorly reflective mass.(17). In our study we found 1 case of rhabdomyosarcoma in a 15 years old female who came with complaints of proptosis since 2 years. The lesion appeared irregular in shape with cystic area containing echoes within. It did not show any vascularity on Doppler imaging. On histopathological evaluation the lesion was found to have undergone cystic degeneration.

7) Haemangioma: Orbital haemangioma may be of cavernous type or capillary type. Cavernous haemangioma is the commonest benign orbital tumour in adults. The majority are intraconal in location and may occasionally impinge on the optic nerve but cause little proptosis. (17). In our study, 2 cases of orbital haemangioma were identified as well circumscribed echogenic masses abutting the globe with internal vascularity on Doppler imaging which corroborate the findings noted in other studies. (11)

8) Metastases: Orbital metastases are uncommon but when present they may produce orbital symptoms prior to the diagnosis of primary malignancy. The orbital metastases maybe from an adjacent primary sinonasal malignancy or from breast, prostate, thyroid, gastrointestinal or renal malignancy. (18). In present study 2 cases of metastases to eye were seen out of which one case was of nasopharyngeal carcinoma and other was of follicular carcinoma of thyroid. Both cases came with painful loss of vision and proptosis. On ultrasound both the lesions were seen in extraocular locations and appeared hypoechoic with high internal vascular flow on colour doppler imaging.

\section{CONCLUSIONS}

In conclusion a total of 15 cases with orbital masses were diagnosed preoperatively amongst 316 patients. Most of the malignant masses appear acoustically solid and hypoechoic with internal vascularity on colour doppler imaging. Most of the benign orbital masses were either solid or cystic with no internal vascularity on colour doppler imaging except for the haemangiomas. The retinoblastomas also show numerous areas of internal calcification causing acoustic shadowing. Most of the patients presented with pain and loss of vision with proptosis. All the identified masses were unilateral lesions. The study thus emphasizes the utility of ultrasound evaluation of orbits prior to surgical interventions for better localisation and characterisation of lesion. It can also act as useful adjunct to higher imaging modalities such as MRI and CT for assessing post-operative prognosis of the patient. 


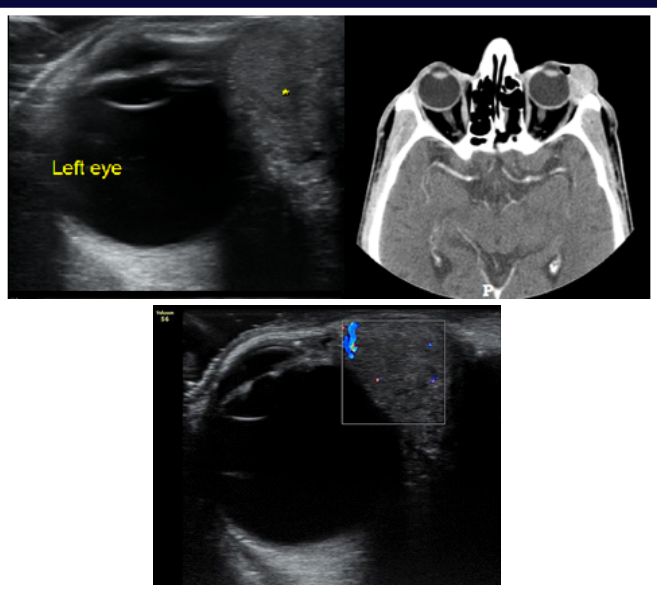

Image 1: Pleomorphic adenoma Hypoechoic solid mass compressing on globe. Doppler shows minimal vascularity in the mass. CT scan shows moderately enhancing lacrimal gland mass

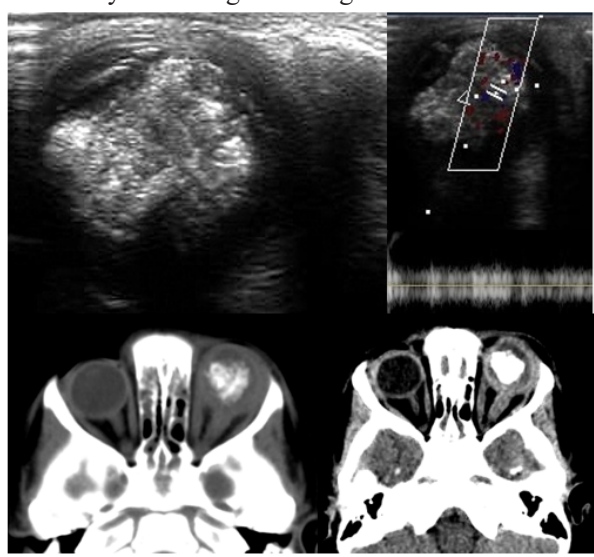

Image 2: Retinoblastoma: USG shows intraocular calcified mass showing vascularity on Doppler imaging. CT shows thickening of retrobulbar part of optic nerve suggestive of optic nerve involvement.
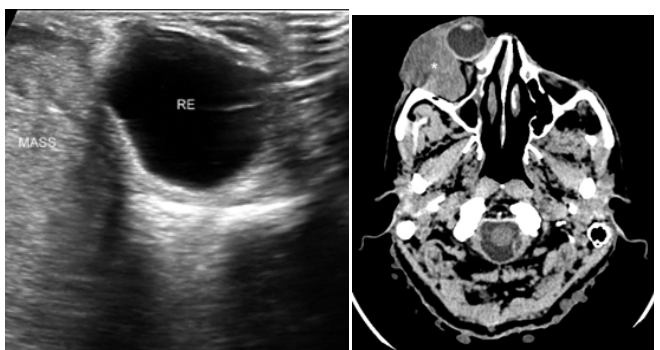

Image 3: Adenoid Cystic Carcinoma of Lacrimal Gland: USG shows heterogeneous echotextures mass compressing the right eyeball. CT shows enhancing right lacrimal gland mass causing proptosis.

\section{REFERENCES}

(Who) WHO. Global data on visual impairements 2010. 2012; Available from: http://www.who.int/blindness/GLOBALDATAFINAL forweb pdf. Accessed 2 Dec 2014

2. Salman A, Parmar P, Vanila CG, Thomas PA, Jesudasan NCA. Is ultrasonography essential before surgery in eyes with advanced cataracts? J Postgrad Med. 2006;52(1):19-22.

3. Garg J, Tirkey E, Jain S, Lakhtakia S, Tiwari A. B - Scan Ultrasonography Before Surgery I N Eyes With Advanced Cataracts: a Useful Prognostic Tool. J Evol Med Dent Sci. 2015;4(37):6372-7.

4. Orbital space-occupying lesions: the evaluation of clinical, radiological and pathological results. Med Bull Sisli Etfal Hosp. 2017:51(4):322-8

5. Aburn NS, Sergott RC. Orbital colour Doppler imaging. Eye. 1993; 7 (Pt 5):639-47.

6. Liao S-L, Lin LL-K. Clinical Applications of Color Doppler Imaging in Orbital Diseases. J Med Ultrasound. 2004;12(2):43-8.

7. Chaudhari HD, Thakkar GN, Gandhi VS, Darji PJ. Role of ultrasonography in evaluation of orbital lesions . 2013;68(22)

8. Sharma O. Orbital sonography with it's clinico-surgical correlation. Indian J Radiol Imaging. 2005;15(April):537.

9. Tailor TD, Gupta D, Dalley RW, Keene CD, Anzai Y. Orbital Neoplasms in Adults: Clinical, Radiologic, and Pathologic Review. RadioGraphics. 2013 Oct 1;33(6):1739-58.

10. Tang W, Hei Y, Xiao L. Recurrent orbital space-occupying lesions: a clinicopathologic study of 253 cases. Chin J Cancer Res. 2013;25(4):423-9.
11. Nagaraju RM. Efficacy of High Frequency Ultrasound in Localization and Characterization of Orbital Lesions. J Clin Diagnostic Res. 2015;9(November 2011):1-6.

12. Kabra R. Ultrasound biomicroscopy as an important diagnostic adjunct in the management of limbal tumors. Int J Curr Res Rev. 2014;6(21):35-40.

13. Aghai G, Fereidoonian NA, Khalatbary MR, Hejazian E, Falavarjani KG. Orbitofrontal Cholesterol Granuloma : Report of Two Cases and Review of Literature. 2011;21-5.

14. Aironi V, Chougule S, Khetarpal S, Bhati G. Retinoblastoma: A spectrum of manifestations in three cases on B-scan. Indian J Radiol Imaging. 2007 Jan $1 ; 17(1): 11-4$

15. Bhatt D. Ultrasonography of ocular tumors. Indian J Radiol Imaging. 2007:17(1):15-6.

16. Silva D. Orbital Tumors. Am J Ophthalmol, 1968 Mar 1;65(3):318-39.

17. A. J. The eye and orbit. In: Fielding IN. CD, editor. Clinical Ultrasound. 2001. p. 659-95.

18. Char DH, Miller T, Kroll S. Orbital metastases: diagnosis and course. Br J Ophthalmol. 1997 May 1;81(5):386 LP-390. 